\title{
Crescimento, acúmulo de nutrientes e fixação biológica de nitrogênio de Flemingia macrophylla ${ }^{1}$
}

\author{
Alexandre Porto Salmi ${ }^{2}$, Ilzo Artur Moreira Risso ${ }^{3}$, José Guilherme Marinho Guerra ${ }^{4}$, Segundo Urquiaga, \\ Adelson Paulo de Araújo ${ }^{6}$ e Antônio Carlos de Souza Abboud ${ }^{7}$
}

\section{RESUMO}

Flemingia macrophylla é uma leguminosa arbustiva asiática, pouco conhecida no Brasil. Este trabalho objetivou avaliar a produção de massa seca, o acúmulo de nutrientes e a fixação biológica de nitrogênio $\left(\mathrm{N}_{2}\right)$ de flemingia. Conduziram-se dois experimentos de campo, entre dezembro de 2006 e dezembro de 2007, em Seropédica, RJ, e em Avelar, RJ. O delineamento experimental utilizado em ambos os experimentos foi de blocos casualizados, com quatro repetições, e os tratamentos foram as diferentes épocas de amostragens. A área foliar, a produção de matéria seca e o acúmulo de nutrientes da parte aérea foram determinados em 12 coletas mensais. A contribuição da fixação biológica de $\mathrm{N}_{2}$ foi estimada pelo método da abundância natural de ${ }^{15} \mathrm{~N}$. A massa seca da parte aérea atingiu $4,0 \mathrm{Mg} \mathrm{ha}^{-1} \mathrm{em}$ Seropédica e 2,3 $\mathrm{Mg} \mathrm{ha}^{-1} \mathrm{em}$ Avelar, aos 360 dias após transplante (DAT). O menor crescimento em Avelar foi associado ao inverno mais frio e seco, em relação a Seropédica. A taxa de crescimento da cultura atingiu valor máximo aos 165 DAT, na primeira floração. O acúmulo de N, P, K, Ca e Mg na parte aérea foi de, respectivamente, 74, 5, 33, 25 e 8 kg ha-1 aos 360 DAT. A percentagem de N, proveniente da fixação biológica de $\mathrm{N}_{2}$ em flemingia, foi de aproximadamente $76 \%$, o que propiciou $57 \mathrm{~kg} \mathrm{ha}^{-1}$ de $\mathrm{N}$ oriundo da fixação biológica, na parte aérea, aos 360 DAT. Os resultados indicam que flemingia é uma espécie que pode contribuir com quantidades significativas de biomassa e nutrientes, especialmente $\mathrm{N}$, sendo promissora para uso como adubo verde e em cultivos em aleias, em sistemas de produção.

Palavras-chave: leguminosa, abundância natural de ${ }^{15} \mathrm{~N}$, análise de crescimento vegetal.

\section{ABSTRACT}

\section{Growth, nutrient accumulation and biological nitrogen fixation of Flemingia macrophylla}

Flemingia macrophylla is an Asian shrub legume scarcely known in Brazil. The objective of this work was to evaluate the growth, nutrient accumulation and biological $\mathrm{N}_{2}$ fixation of flemingia. Two field experiments were conducted from December 2006 to December 2007, in Seropédica and in Avelar, Rio de Janeiro State. Both experiments were arranged in a completely randomized block design with four repetitions, and the treatments consisted of the different sampling times. Leaf area, biomass and nutrient accumulation in leaves and branches were measured in twelve monthly samplings. Biological $\mathrm{N}_{2}$ fixation was estimated by the natural abundance of ${ }^{15} \mathrm{~N}$. Shoot biomass yield reached $4.0 \mathrm{Mg}$ ha $^{-1}$ in Seropédica and 2.3 Mg ha ${ }^{1}$ in Avelar at 360 days after transplanting (DAT). The lower growth in Avelar was associated with the colder and drier winter than in Seropédica. The highest crop growth rate was observed at 165 DAT,

\footnotetext{
Recebido para publicação em 06/10/2011 e aprovado em 04/01/2013

${ }^{1}$ Parte da Dissertação de Mestrado do primeiro autor no Curso de Pós-Graduação em Fitotecnia da UFRRJ. Apoio financeiro Fundação Carlos Chagas de Amparo à Pesquisa do Estado do RJ (FAPERJ)

${ }^{2}$ Engenheiro-Agrônomo, Doutor. Departamento de Fitotecnia, Universidade Federal Rural do Rio de Janeiro, 23890-000, Seropédica, Rio de Janeiro, Brasil. salmialexandre@ig.com.br (autor para correspondência)

${ }^{3}$ Licenciado em Ciências Agrícolas, Mestre. Área de Comunicação e Negócios da Embrapa Agrobiologia, Rodovia BR 465, Km 7, Seropédica, Rio de Janeiro, Brasil. ilzo@cnpab.embrapa.br

${ }^{4}$ Engenheiro-Agrônomo, Ph.D. Embrapa Agrobiologia, Rodovia BR 465, Km 7, Seropédica, Rio de Janeiro, Brasil. gmguerra@cnpab.embrapa.br

${ }_{5}^{5}$ Engenheiro-Agrônomo, Ph.D. Embrapa Agrobiologia, Rodovia BR 465, Km 7, Seropédica, Rio de Janeiro, Brasil. urquiaga@ cnpab.embrapa.br

${ }^{6}$ Engenheiro-Agrônomo, Ph.D. Departamento de Solos, Universidade Federal Rural do Rio de Janeiro, 23890-000, Seropédica, Rio de Janeiro, Brasil. aparaujo@ufrrj.br

Engenheiro-Agrônomo, Ph.D. Departamento de Fitotecnia, Universidade Federal Rural do Rio de Janeiro, 23890-000, Seropédica, Rio de Janeiro, Brasil. abboud@ufrrj.br
} 
at the first flowering. Accumulation of N, P, K, Ca and Mg in shoots was, respectively, 74, 5, 33, 25 and $8 \mathrm{~kg} \mathrm{ha}^{-1}$ at 360 DAT. The percentage of $\mathrm{N}$ derived from biological $\mathrm{N}_{2}$ fixation was approximately $76 \%$ which provided $57 \mathrm{~kg} \mathrm{ha}^{-1}$ of $\mathrm{N}$ originated from symbiosis in shoots at $360 \mathrm{DAT}$. The results indicate that flemingia may provide significant amounts of biomass and nutrients, especially $\mathrm{N}$, arising as a promising species to be introduced as green manure and alley cropping in agricultural systems.

Key words: shrub legume, ${ }^{15} \mathrm{~N}$ natural abundance, plant growth analysis.

\section{INTRODUÇÃO}

A diversificação dos sistemas agrícolas, tanto no tempo, quanto no espaço, pode ser alcançada por meio de rotações de culturas, policultivos, sistemas agro florestais, cultivo em aleias, integração lavoura-pecuária, dentre outras práticas. Neste contexto, a adubação verde consiste na utilização de plantas, em particular da família das leguminosas, em rotação ou consórcio com as culturas de interesse econômico, reduzindo o uso de nitrogênio $(\mathrm{N})$ por via da adubação mineral, contribuindo para a ciclagem de nutrientes e o aumento da diversidade vegetal (Guerra et al., 2003). Algumas espécies de leguminosas podem aportar elevadas quantidades de $\mathrm{N}$ aos sistemas de produção, uma vez que se associam com bactérias que fixam o $\mathrm{N}_{2}$ atmosférico, podendo proporcionar aumentos de produtividade das culturas consorciadas ou em sucessão (Oliveira et al., 2006). Além disso, o uso de adubos verdes pode trazer outros benefícios, tais como redução de processos erosivos, redução do aquecimento excessivo do solo, manutenção da fertilidade e da umidade favorável à atividade biológica, supressão de plantas invasoras, adição de matéria orgânica e melhoria da estrutura dos solos (Espíndola et al., 2006).

A introdução de leguminosas arbóreas, em sistemas agro florestais e silvipastoris, pode aumentar a sustentabilidade agrícola, por meio da restauração e manutenção da fertilidade do solo, do combate à erosão e à desertificação, e do fornecimento de energia, como a lenha (Machado $e t$ al., 2012). Entretanto, a estimativa da contribuição da fixação biológica de $\mathrm{N}_{2}$ em espécies perenes é particularmente difícil. A técnica da abundância natural de ${ }^{15} \mathrm{~N}$, a qual se baseia no ligeiro enriquecimento de ${ }^{15} \mathrm{~N}$ observado nos solos em relação à atmosfera, está menos sujeita a erros advindos de variações temporais na marcação isotópica do solo, mas demanda a comparação da composição de ${ }^{15} \mathrm{~N}$ da espécie fixadora com uma espécie controle não fixadora, com o mesmo padrão de absorção de $\mathrm{N}$ do solo (Unkovich \& Pate, 2000). Apesar disto, a abundância natural de ${ }^{15} \mathrm{~N}$ apresenta-se como a técnica mais promissora para a estimativa da fixação biológica de $\mathrm{N}_{2}$, em espécies perenes, em condições de campo (Boddey et al., 2000).
A espécie Flemingia macrophylla (syn. Flemingia congesta), leguminosa com centro de origem na Ásia, é uma planta perene de porte arbustivo, que pode atingir 4 $\mathrm{m}$ de altura. Em alguns países, essa espécie tem-se mostrado promissora para uso como adubo verde em diversos sistemas produtivos, podendo formar faixas para o cultivo em aleias, proporcionando sombra em plantações consorciadas e proteção do solo, além de poder ser utilizada para alimentação animal e produção de lenha. A flemingia apresenta vantagens adicionais, como a adaptação a uma ampla gama de solos, incluindo aqueles com baixa fertilidade e alta acidez, a tolerância à seca e a boa capacidade de rebrota após o corte (Andersson et al., 2006). Banful et al. (2000) relataram que a espécie apresentou elevada produção de biomassa, $35 \mathrm{Mg} \mathrm{ha}^{-1}$, na soma de seis cortes anuais, quando conduzidas em aleias, em Gana, na África, e pode inclusive atuar no controle de nematoides do solo. Desta forma, tornam-se necessários estudos para avaliar a adaptação de flemingia no Brasil e seu potencial para produção de biomassa e acúmulo de nutrientes. Objetivou-se, com este trabalho, avaliar a produção de massa seca, o acúmulo de nutrientes e a fixação biológica de $\mathrm{N}_{2}$ de Flemingia macrophylla.

\section{MATERIAL E MÉTODOS}

Foram conduzidos dois experimentos de campo, em localidades diferentes. O primeiro experimento foi conduzido entre os meses de janeiro e dezembro de 2007, em um Planossolo Háplico, na Embrapa Agrobiologia, no município de Seropédica (RJ), situado na latitude $22^{\circ} 45^{\prime} \mathrm{S}$, longitude $43^{\circ} 41^{\prime}$ 'W e altitude de $33 \mathrm{~m}$. O clima da região é tropical com estação seca de inverno (Aw na classificação de Köppen). As análises químicas do solo (Embrapa, 1997) indicaram, na camada de 0-20 cm: $12,9 \mathrm{~g} \mathrm{~kg}^{-1} \mathrm{de} \mathrm{C}, \mathrm{pH}$ em água 5,6, $1,3 \mathrm{cmol}_{c} \mathrm{dm}^{-3} \mathrm{de} \mathrm{Ca}, 1,0 \mathrm{cmol}_{c} \mathrm{dm}^{-3} \mathrm{de} \mathrm{Mg}, 0 \mathrm{cmol}_{c}$ $\mathrm{dm}^{-3} \mathrm{de} \mathrm{Al}, 0,37 \mathrm{cmol}_{\mathrm{c}} \mathrm{dm}^{-3} \mathrm{de} \mathrm{K}, 5 \mathrm{mg} \mathrm{dm}^{-3}$ de P disponível; e na camada de 20-40 cm: 7,9 $\mathrm{g} \mathrm{kg}^{-1} \mathrm{de}$ C, $\mathrm{pH}$ em água 5,3, 0,9 $\mathrm{cmol}_{\mathrm{c}} \mathrm{dm}^{-3} \mathrm{de} \mathrm{Ca}, 0,7 \mathrm{cmol}_{\mathrm{c}} \mathrm{dm}^{-3} \mathrm{de} \mathrm{Mg}, 0,3 \mathrm{cmol}_{\mathrm{c}} \mathrm{dm}^{-3} \mathrm{de} \mathrm{Al}$, $0,07 \mathrm{cmol}_{\mathrm{c}} \mathrm{dm}^{3} \mathrm{de} \mathrm{K}, 3 \mathrm{mg} \mathrm{dm}^{-3} \mathrm{de}$ P disponível.

$\mathrm{O}$ segundo experimento foi conduzido entre os meses de janeiro e dezembro de 2007, em um Argissolo Verme- 
lho-Amarelo franco argilo-arenoso, no Campo Experimental da Empresa de Pesquisa Agropecuária do Estado do Rio de Janeiro - PESAGRO-RIO, em Avelar, município de Paty do Alferes (RJ), localizada a $22^{\circ} 21^{\prime} \mathrm{S}, 43^{\circ} 25^{\prime} \mathrm{W}$ e altitude de $507 \mathrm{~m}$. O clima na região é tropical úmido de altitude (Cwb na classificação de Köppen). As análises químicas do solo indicaram, na camada de 0-20 cm: 8,3 g $\mathrm{kg}^{-1}$ de C, pH em água 4,9, 2,5 $\mathrm{cmol}_{\mathrm{c}} \mathrm{dm}^{-3} \mathrm{de} \mathrm{Ca}, 2 \mathrm{cmol}$ c $\mathrm{dm}^{-3}$ de $\mathrm{Mg}, 0,5 \mathrm{cmol}_{\mathrm{c}} \mathrm{dm}^{-3}$ de Al, 0,79 $\mathrm{cmol}_{\mathrm{c}} \mathrm{dm}^{-3} \mathrm{de} \mathrm{K}, 4$ $\mathrm{mg} \mathrm{dm}{ }^{-3}$ de $\mathrm{P}$ disponível; e na camada de 20-40 cm: 5,6 g $\mathrm{kg}^{-1} \mathrm{de} \mathrm{C}$, pH em água 4,8, 1,9 $\mathrm{cmol}_{\mathrm{c}} \mathrm{dm}^{-3} \mathrm{de} \mathrm{Ca}, 1,3 \mathrm{cmol}$ $\mathrm{dm}^{-3} \mathrm{de} \mathrm{Mg}, 0,7 \mathrm{cmol}_{\mathrm{c}} \mathrm{dm}^{-3} \mathrm{de} \mathrm{Al}, 0,39 \mathrm{cmol}_{\mathrm{c}} \mathrm{dm}^{-3} \mathrm{de} \mathrm{K}, 16$ $\mathrm{mg} \mathrm{dm}^{-3}$ de $\mathrm{P}$ disponível.

Os dados meteorológicos do período experimental foram obtidos nos Postos Agrometeorológicos das Estações Experimentais de Seropédica-RJ e de Avelar-RJ (PESAGRO-RIO/INMET) (Figura 1).

Para a superação da dormência, as sementes de flemingia foram imersas em água quente $\left(90^{\circ} \mathrm{C}\right)$, seguido do resfriamento por uma hora (Salmi et al., 2008). As mudas foram produzidas em casa de vegetação, em bandejas de isopor de 72 células, com duas sementes por célula. $\mathrm{O}$ substrato utilizado foi uma mistura composta por $83 \%$ de

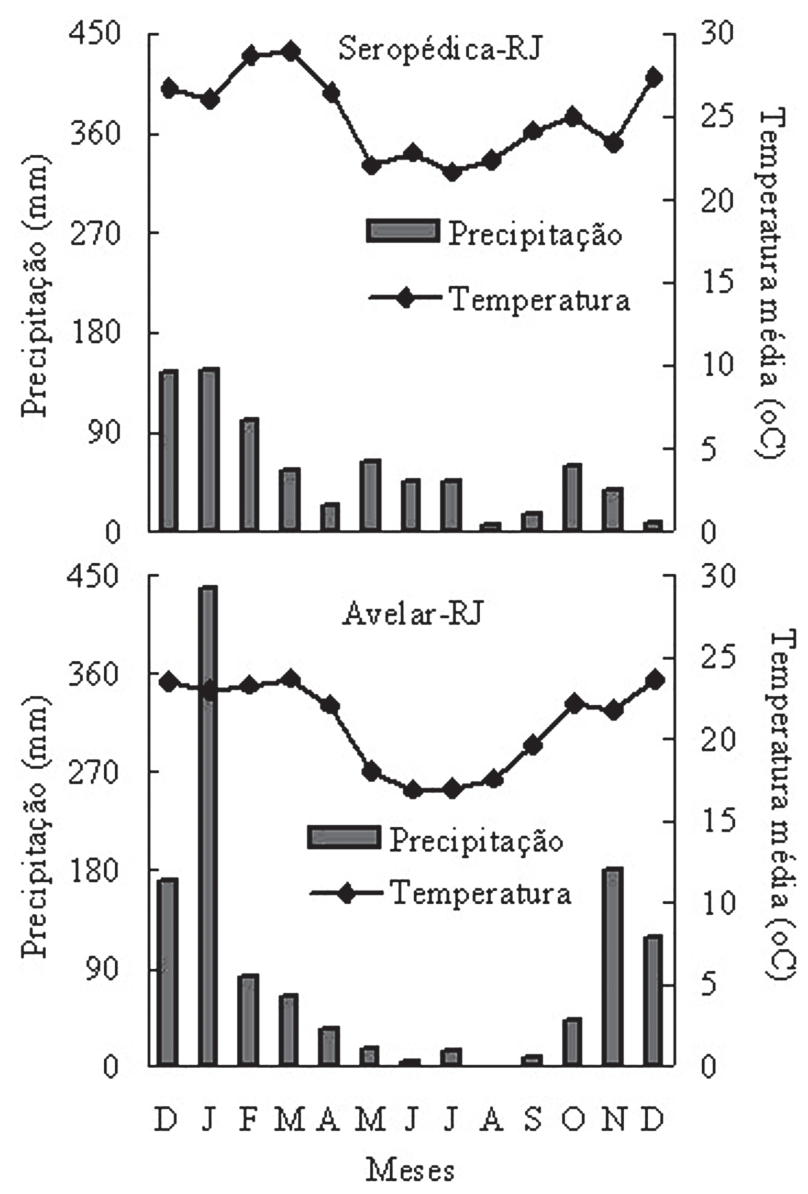

Figura 1. Precipitação pluvial e temperatura média nos municípios de Seropédica-RJ e Avelar-RJ, durante os meses de dezembro de 2006 a dezembro de 2007. vermicomposto, $15 \%$ de fino de carvão vegetal e $2 \%$ de torta de mamona. Encontrou-se, na caracterização do substrato utilizado na produção das mudas, $5,46 \mathrm{~g} \mathrm{~kg}^{-1} \mathrm{de}$ $\mathrm{C}$, pH em água 6,6, $12 \mathrm{cmol}_{\mathrm{c}} \mathrm{dm}^{-3} \mathrm{de} \mathrm{Ca}, 11,5 \mathrm{cmol}_{\mathrm{c}} \mathrm{dm}^{-3} \mathrm{de}$ $\mathrm{Mg}, 0 \mathrm{cmol}_{\mathrm{c}} \mathrm{dm}^{-3} \mathrm{de} \mathrm{Al}, 0,12 \mathrm{cmol}_{\mathrm{c}} \mathrm{dm}^{-3} \mathrm{de} \mathrm{K}, 18 \mathrm{mg} \mathrm{dm}^{-3} \mathrm{de}$ $\mathrm{P}$ disponível. Efetuou-se o transplante das mudas quando essas apresentavam dois pares de folhas, em dezembro de 2006, com espaçamento de 2,0 m x 0,5 m em um talhão de $35 \mathrm{~m}$ x $35 \mathrm{~m}$.

A adubação realizada no plantio, em ambos os experimentos, foi de $60 \mathrm{~kg} \mathrm{ha}^{-1}$ de $\mathrm{P}_{2} \mathrm{O}_{5}$ e $40 \mathrm{~kg} \mathrm{ha}^{-1}$ de $\mathrm{K}_{2} \mathrm{O}$, baseando-se na recomendação de adubação para Leucaena leucocephala, segundo De-Polli et al. (1988). Utilizou-se como fontes desses nutrientes o termofosfato e o cloreto de potássio, respectivamente.

Ambos os experimentos utilizaram delineamento experimental em blocos ao acaso, com quatro repetições. Foram realizadas 12 amostragens das plantas, aos 30, 60, 90, 120, 150, 180, 210, 240, 270, 300, 330 e 360 dias após transplante (DAT). Em cada época, determinou-se a altura das plantas, o número de folíolos da planta inteira, o diâmetro da base do caule, com auxílio de um paquímetro, e o número de ramificações.

No experimento em Seropédica, duas plantas de cada parcela, nas diferentes épocas de amostragem, foram cortadas ao nível do solo e separadas em folhas e ramos. Os folíolos foram separados para determinação da área foliar, por meio do medidor fotoelétrico (LI-COR 3100). A parte aérea das plantas foi secada em estufa, a $65^{\circ} \mathrm{C}$, pesada e moída, analisando-se os teores de N, P, K, Ca e Mg (Malavolta et al., 1997). O acúmulo de nutrientes foi calculado pelo produto entre o teor e a matéria seca. No município de Avelar, aos 360 DAT, foi efetuado o corte da parte aérea de seis plantas de cada parcela e o material foi secado em estufa, a $65^{\circ} \mathrm{C}$, para a determinação da matéria seca da parte aérea.

Dentre as funções de crescimento testadas para ajuste dos dados no tempo por meio de regressão, foi escolhido o modelo de Gompertz, W = a exp(-b exp(-cT)), para matéria seca da parte aérea, área foliar e acúmulos de nutrientes, considerando-se o tempo (em dias após transplante) como variável independente. A partir dos modelos ajustados, foram derivadas a taxa de crescimento da cultura, que corresponde à variação, no tempo, da matéria seca por unidade de terreno, e a taxa de assimilação líquida, que corresponde à variação no acúmulo de matéria seca por unidade de área foliar (Hunt, 1982).

A contribuição da fixação biológica de $\mathrm{N}_{2}$ para o acúmulo de $\mathrm{N}$ pela flemingia foi avaliada apenas no experimento conduzido no município de Seropédica. O método utilizado foi o da abundância natural de ${ }^{15} \mathrm{~N}$ (Unkovich $\&$ Pate, 2000). Foram efetuadas coletas aos 240, 300 e 360 DAT, por meio de uma amostragem composta da parte 
aérea de três plantas de cada bloco experimental. Concomitantemente, foram amostradas na área experimental plantas de capim elefante (Pennisetum purpureum Schum.), utilizada como testemunha, planta não fixadora de nitrogênio atmosférico. Os materiais secos foram moídos e analisados quanto à abundância natural de ${ }^{15} \mathrm{~N}$ $\left(\delta^{15} \mathrm{~N}\right)$, utilizando-se o espectrômetro de massa (Finnigan DeltaPlus, Finnigan MAT, Germany).

O percentual de $\mathrm{N}$ derivado da atmosfera (\%Ndfa) foi calculado por (Unkovich \& Pate, 2000):

$\% \mathrm{Ndfa}=\left[\left(\delta^{15} \mathrm{~N}\right.\right.$ testemunha $-\delta^{15} \mathrm{~N}$ leguminosa $) /\left(\delta^{15} \mathrm{~N}\right.$ testemunha-B)] x 100,

sendo $\delta^{15} \mathrm{~N}$ testemunha o valor de $\delta^{15} \mathrm{~N}$ da planta não fixadora, utilizada como referência, $\delta^{15} \mathrm{~N}$ leguminosa o valor de $\delta^{15} \mathrm{~N}$ de flemingia, e, B, o valor da discriminação isotópica de ${ }^{15} \mathrm{~N}$, feita pela leguminosa durante o processo de FBN, empregando-se o valor de -0,9 estimado para Cajanus cajan. A quantidade de $\mathrm{N}$ oriundo da fixação de $\mathrm{N}_{2}$ foi obtida pelo produto entre a $\% \mathrm{Ndfa}$ e quantidade de $\mathrm{N}$ total acumulado na parte aérea (Boddey et al., 2000). Os mesmos autores destacam que, como a abundância natural de ${ }^{15} \mathrm{~N}$ varia nos diferentes tecidos da planta e durante a ontogenia vegetal, é recomendável a amostragem da planta inteira, ou, ao menos, de toda a parte aérea, para estimar a fixação de $\mathrm{N}_{2}$ por esta técnica, como efetuado no presente trabalho.

Foi efetuada a análise de variância para os dados de $\mathrm{N}$ acumulado e da contribuição da fixação de $\mathrm{N}_{2}$, sendo as médias de cada época de coleta comparadas pelo teste de Tukey, a 5\% de probabilidade.

\section{RESULTADOS E DISCUSSÃO}

Aos 360 dias após o transplante (DAT), as plantas de flemingia, conduzidas em Seropédica, apresentavam $174 \mathrm{~cm}$ de altura (Figura 2A). Em Avelar, as plantas atingiram a altura de $155 \mathrm{~cm}$. Burguer \& Brasil (1986) registraram altura de $125 \mathrm{~cm}$ de flemingia, aos 190 DAT no Pará, similar à observada em Seropédica. Andersson et al. (2006) relatam variações de altura de 40 a $287 \mathrm{~cm}$, entre acessos de germoplasma de flemingia, em plantas com 14 meses, na Colômbia. O diâmetro da base do caule atingiu $18 \mathrm{~mm}$ e $15 \mathrm{~mm}$ aos 360 DAT, em Seropédica e Avelar, respectivamente (Figura 2B), o que confirma o aspecto semilenhoso da espécie, relatado por Andersson et al. (2006). Os autores ressaltam, ainda, o potencial da espécie para uso como lenha, em pequena escala. O número de folíolos foi superior, em Seropédica, em relação a Avelar (Figura 2C). Nos meses de julho a setembro de 2007, por causa da ocorrência de um período seco, com déficit hídrico em ambos os locais (Figura 1), houve senescência de folhas mais velhas e redução do número de folíolos, a partir de 210 DAT, em Seropédica, e de 120 DAT, em Avelar. Com o início das chuvas, a partir de setembro, em ambos os locais (Figura 1), ocorreu aumento progressivo do número de folíolos, a partir dos 300 DAT. A espécie apresentou elevado número de ramificações (Figura 2D), originadas desde a base do caule da planta, com gemas meristemáticas provenientes de partes radiculares mais próximas do colo. Fato que confirma a boa capacidade de ramificação da espécie, relatada por Andersson et al. (2006).

De forma geral, as variáveis avaliadas não apresentaram grandes diferenças entre os locais de cultivo, até os 120 DAT; porém, a partir de 150 DAT, até ao final da condução dos experimentos, as plantas em Seropédica mostraram melhor crescimento em relação às plantas em Avelar (Figura 2). O município de Avelar apresentou um inverno mais frio e seco (Figura 1), o que, provavelmente limitou o crescimento e retardou sua retomada a partir da primavera (300 DAT). Isto indica que o uso da flemingia como adubo verde pode ser restrito, em regiões de clima mais frio e seco. Andersson et al. (2006) sugerem uma seleção de genótipos de $F$. macrophylla, mais tolerantes a condições de baixas temperatura e pluviosidade.

A produção de matéria seca da parte aérea das plantas de flemingia, em Seropédica, atingiu $405 \mathrm{~g} \mathrm{~m}^{-2}$, aproximadamente 4,0 $\mathrm{Mg} \mathrm{ha}^{-1}$ aos 360 DAT (Figura 3A), valor superior ao da produção em Avelar, que atingiu 2,3 $\mathrm{Mg} \mathrm{ha}^{-1}$. A espécie apresentou uma relação entre matéria seca de ramos e folhas de 2:1, em ambos os locais.

Como mencionado para o número de folíolos, o índice de área foliar também aumentou até os 240 DAT, decrescendo entre 240 e 300 DAT, atingindo $1,8 \mathrm{~m}^{2} \mathrm{~m}^{-2}$ aos 360 DAT (Figura 3B). A taxa de crescimento da cultura atingiu valor máximo aos 165 DAT, em torno de $1,54 \mathrm{~g} \mathrm{~m}^{-2} \mathrm{dia}^{-1}$ (Figura 3C). Este valor máximo da taxa de crescimento da cultura coincide com a primeira floração, entre os 150 e 180 DAT. A taxa de assimilação líquida foi máxima no início do ciclo, declinando com o tempo (Figura 3D), com valores menores por ocasião da segunda florada da espécie, entre os 330 e 360 DAT. De acordo com Hunt (1982), quando as plantas passam a designar os fotoassimilados para os frutos e sementes, geralmente ocorre uma diminuição da taxa de assimilação líquida. Os valores da taxa de assimilação líquida são similares aos observados em espécies de metabolismo C3, neste estádio ontogenético (Hunt, 1982), indicando uma boa adaptação de flemingia às condições locais.

Houve um aumento da quantidade acumulada de nutrientes na parte aérea nas plantas de flemingia ao longo do tempo, atingido $81 \mathrm{~kg} \mathrm{ha}^{-1} \mathrm{de} \mathrm{N}, 5 \mathrm{~kg} \mathrm{ha}^{-1} \mathrm{de} \mathrm{P}$, $33 \mathrm{~kg} \mathrm{ha}^{-1} \mathrm{de} \mathrm{K}, 25 \mathrm{~kg} \mathrm{ha}^{-1}$ de Ca e $8 \mathrm{~kg} \mathrm{ha}^{-1} \mathrm{de} \mathrm{Mg}$, aos 360 DAT, em Seropédica (Figura 4). Brasil (1992) en- 

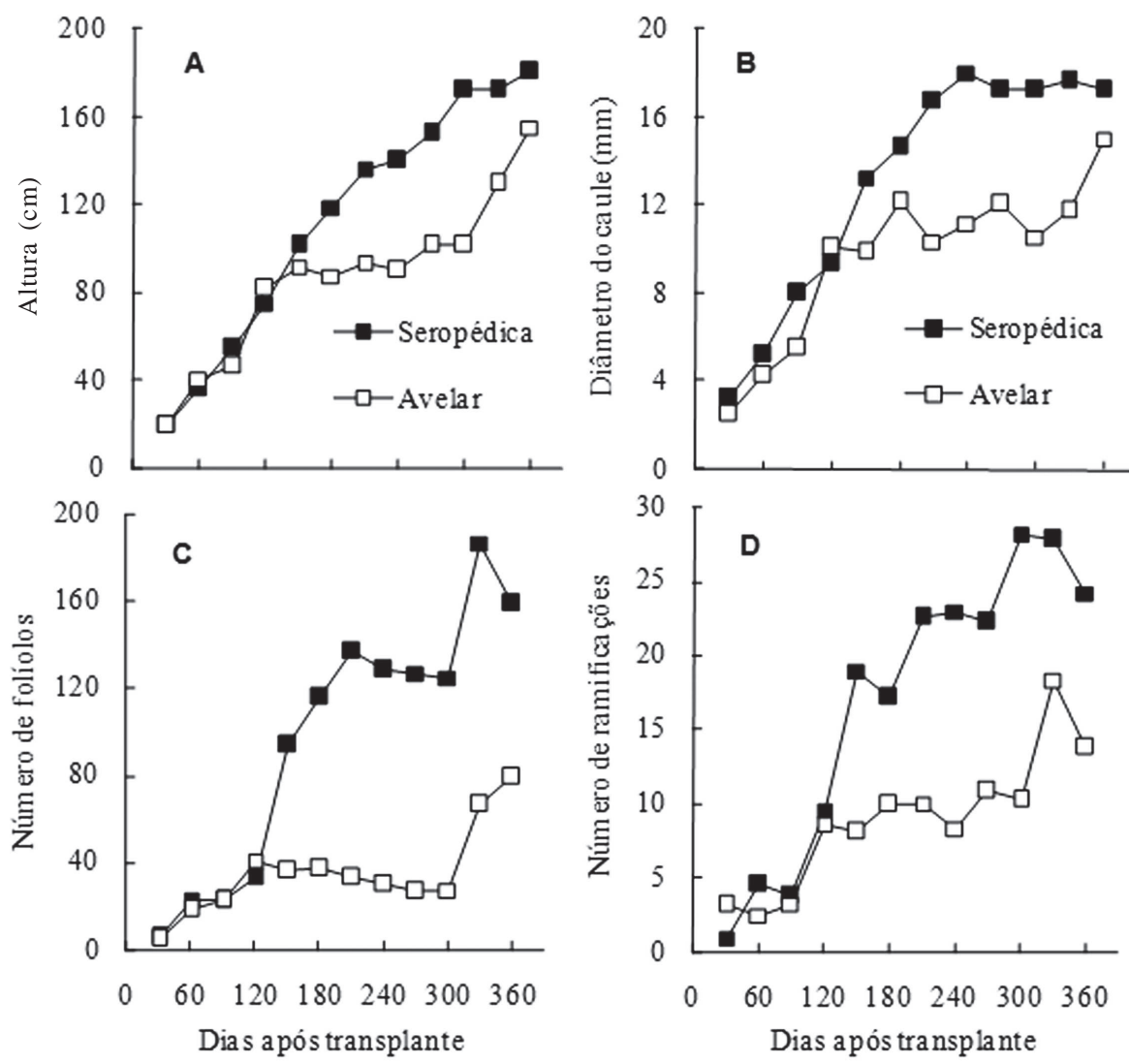

Figura 2. Altura da planta (A), diâmetro da base do caule (B), número de folíolos (C) e número de ramificações (D), durante o ciclo de crescimento de plantas de Flemingia macrophylla, avaliados nos municípios de Seropédica-RJ (ロ) e Avelar-RJ (ם).
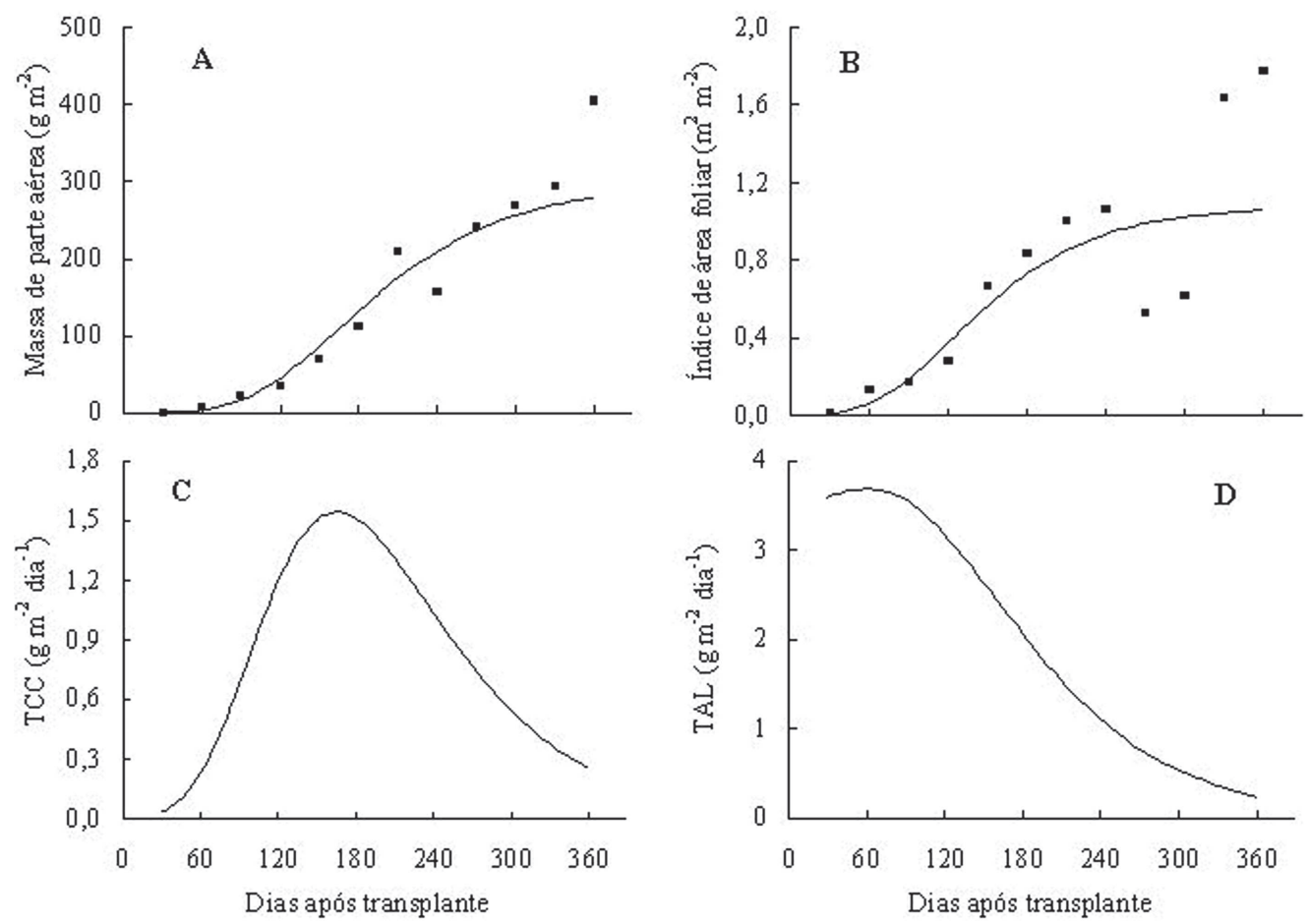

Figura 3. A: Massa seca de parte aérea; B: índice de área foliar; C: taxa de crescimento da cultura (TCC) e D: taxa de assimilação líquida (TAL) de Flemingia macrophylla durante o ciclo de crescimento no município de Seropédica-RJ. Os símbolos representam as médias experimentais, e a linha, o modelo de Gompertz ajustado aos dados primários. Modelos ajustados, com o tempo T (em dias) como variável independente: massa seca $=2988 \exp (-10,154 \exp (-0,01402 \mathrm{~T})) \mathrm{R}^{2}$ 0,968; área foliar $=10806 \exp (-7,7134 \exp (-$ $0,01659 \mathrm{~T})) \mathrm{R}^{2} 0,876$. 

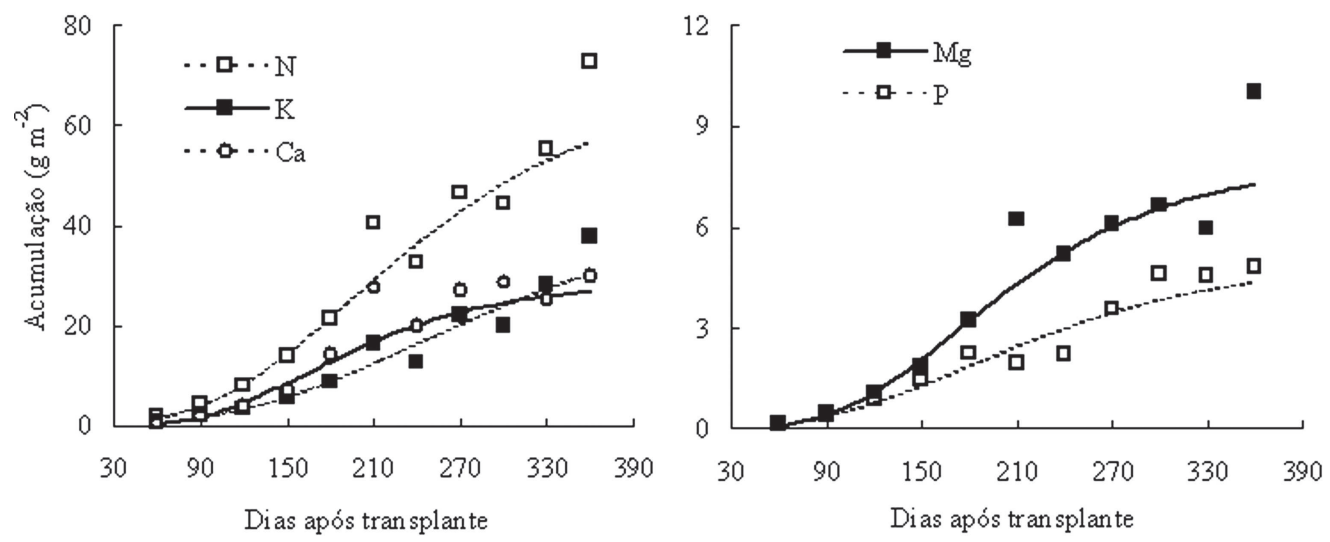

Figura 4. Acúmulo de nutrientes $\left(\mathrm{g} \mathrm{m}^{-2}\right)$ durante o ciclo de crescimento das plantas de Flemingia macrophylla, no município de Seropédica-RJ. Os símbolos representam as médias experimentais, e as linhas, o modelo de Gompertz ajustado aos dados primários. Modelos ajustados com o tempo $\mathrm{T}$ (em dias) como variável independente: $\mathrm{N}=687,8 \exp (-7,0149 \exp (-0,00999 \mathrm{~T})) \mathrm{R}^{2} 0,909 ; \mathrm{P}=$ $443,3 \exp (-6,8273 \exp (-0,00803 \mathrm{~T})) \mathrm{R}^{2} 0,929 ; \mathrm{Ca}=286,0 \exp (-9,5399 \exp (-0,01383 \mathrm{~T})) \mathrm{R}^{2}$ 0,925; $\mathrm{Mg}=78,9 \exp (-9,6837 \exp (-$ $0,01324 \mathrm{~T})) \mathrm{R}^{2} 0,947 ; \mathrm{P}=50,3 \exp (-6,8331 \exp (-0,01072 \mathrm{~T})) \mathrm{R}^{2} 0,907$.

Tabela 1. Total de N acumulado e contribuição da fixação biológica de nitrogênio (FBN) na parte aérea de Flemingia macrophylla em três épocas de crescimento no município de Seropédica-RJ

\begin{tabular}{lccc}
\hline Dias após transplante & $\begin{array}{c}\text { Quantidade total de } \mathbf{N} \\
\left(\mathbf{k g ~ h a}^{-\mathbf{1}}\right)\end{array}$ & $\begin{array}{c}\text { Contribuição da FBN } \\
(\mathbf{\%})\end{array}$ & $\begin{array}{c}\text { Quantidade de N fixado } \\
\left(\mathbf{k g ~ h a}^{-1}\right)\end{array}$ \\
\hline 240 & $32,5 \mathrm{~b} \mathbf{b}^{*}$ & $78,8 \mathrm{a}$ & $25,6 \mathrm{~b}$ \\
300 & $34,4 \mathrm{~b}$ & $76,0 \mathrm{a}$ & $26,2 \mathrm{~b}$ \\
360 & $75,0 \mathrm{a}$ & $75,9 \mathrm{a}$ & $56,9 \mathrm{a}$ \\
\hline C.V. $(\%)$ & 19,8 & 6,6 & 19,0 \\
\hline
\end{tabular}

*Médias seguidas da mesma letra na coluna não diferem entre si pelo teste de Tukey a 5\% de probabilidade.

controu valores similares para o acúmulo de nutrientes em flemingia no Estado do Pará, 74, 4 de N, 4 kg ha ${ }^{-1}$ de P e 50 de $\mathrm{K}$, aos 370 dias após semeadura realizada diretamente a campo.

Para a estimativa da contribuição da fixação biológica de $\mathrm{N}_{2}$, observou-se pequena variação de acordo com as épocas avaliadas (240, 300 e 360 DAT), com valores entre 75,9 e 78,8 \% do $\mathrm{N}$ oriundo da fixação biológica de $\mathrm{N}$ (FBN) (Tabela 1). Peoples et al. (1996) estimaram, por meio da abundância natural de ${ }^{15} \mathrm{~N}$, contribuições da fixação de $\mathrm{N}_{2}$, variando entre 48 e $86 \%$, em leguminosas arbustivas submetidas a podas sucessivas, na Austrália. Além disto, as estimativas da quantidade de $\mathrm{N}$ oriundo da simbiose são dependentes da precisão das mensurações de biomassa e de $\mathrm{N}$ acumulado por leguminosas perenes, tarefa de difícil consecução em condições de campo (Boddey et al., 2000). Cabe destacar que não foi mensurado o aporte de $\mathrm{N}$, da FB presente em raízes e nódulos de flemingia.

A contribuição da fixação biológica de nitrogênio é de $76,9 \%$, equivalente a $57 \mathrm{~kg} \mathrm{ha}^{-1}$ de N, derivado da fixação, aos 360 DAT (Tabela 1). As quantidades de $\mathrm{N}$ fixado por leguminosas perenes são bastante variáveis. Dakora \& Keya (1997) relatam valores entre 43 e $581 \mathrm{~kg} \mathrm{ha}^{-1}$ de $\mathrm{N}$ por ano. Como a contribuição percentual da fixação de $\mathrm{N}_{2}$ foi relativamente estável ao longo do tempo (Tabela 1), a quantidade de $\mathrm{N}$ fixada foi dependente da variação temporal da produção de matéria seca. Isto indica que é possível obter maior aporte de $\mathrm{N}$ da FB com a produção elevada de matéria seca, o que poderia ser alcançado por meio do manejo de podas frequentes, utilizando-se a biomassa da rebrota da espécie. A elevada produção de matéria seca de flemingia, associada ao seu caráter perene, pode potencializar o aporte de $\mathrm{N}$ proveniente da fixação biológica nos sistemas de produção, quando manejada e utilizada para fins de adubação verde.

\section{CONCLUSÕES}

Os valores encontrados de matéria seca e de nutrientes acumulados, bem como a percentagem de $\mathrm{N}$ proveniente da FBN, tornam Flemingia macrophylla uma espécie promissora para uso como adubo verde, em sistemas diversos de produção, mostrando-se interessante fonte de biomassa para o manejo de agroecossistemas tropicais.

\section{AGRADECIMENTOS}

À Fundação Carlos Chagas de Amparo à Pesquisa do Estado do Rio de Janeiro (FAPERJ), pela concessão da bolsa Cientista do Nosso Estado. 


\section{REFERÊNCIAS}

Andersson MS, Schultze-Kraft R, Cansa M, Hincapie B \& Lascano CE (2006) Morphological, agronomic and forage quality diversity of the Flemingia macrophylla world collection. Field Crops Research, 96:387-406.

Banful B, Dzietror A, Ofori I \& Hemeng OB (2000) Yield of plantain alley cropped with Leucaena leucocephala and Flemingia macrophylla in Kumasi, Ghana. Agroforestry Systems, 49:189-199.

Boddey RM, Peoples MB, Palmer B \& Dart P (2000) Use of ${ }^{15} \mathrm{~N}$ abundance technique to quantify biological nitrogen fixation by woody perennials. Nutrient Cycling in Agroecosystems, $57: 235-270$

Burguer D \& Brasil EC (1986) A produção de adubos orgânicos no sistema "cultivo em faixas". In: Embrapa Centro de Pesquisa Agropecuária do Trópico Úmido. Pesquisa sobre utilização e conservação do solo da Amazônia Oriental: relatório final do Convênio Embrapa-CPATU/GTZ. Belém, Embrapa-CPATU/ GTZ, 1986. p. 223-243. (Documentos, 40).

Brasil EC (1992) Mesa redonda sobre recuperação de solos através do uso de leguminosas. Trabalhos e recomendações. Belém, Embrapa-CPATU/GTZ. 131p. (Documentos, 67).

Dakora FD \& Keya SO (1997) Contribution of legume nitrogen fixation to sustainable agriculture in Sub-Saharan Africa. Soi Biological Biochemistry, 29:809-818.

De-Polli H (1988) Manual de adubação para o Estado do Rio de Janeiro. Itaguaí, Universidade Rural. 179p.

Embrapa Centro Nacional de Pesquisa de Solos (1997) Manual de métodos de análise de solo. $2^{\mathrm{a}}$ ed. Rio de Janeiro, Centro Nacional de Pesquisa de Solos. 212p.

Espíndola JAA, Guerra JGM, Perin A, Teixeira MG, Almeida DL, Urquiaga S \& Busquet RNB (2006) Bananeiras consorciadas com leguminosas herbáceas perenes utilizadas como coberturas vivas. Pesquisa Agropecuária Brasileira, 41:415-420.
Guerra JGM, De-Polli H \& Almeida DL de (2003) Managing carbon and nitrogen in tropical organic farming trough green manure. In: Adetola Badejo M, Togun AO (Eds.). Strategies and tactics of sustainable agriculture in the tropics (STASAT). Ibadan, College Press. v.2. p.125-140.

Hunt R (1982) Plant growth curves: the functional approach to plant growth analysis. London, Arnold E. 248p.

Machado FA, Bezerra Neto E, Nascimento M do PSCB, Silva LM, Barreto LP, Nascimento HTS \& Leal JA (2012) Produção e qualidade da serrapilheira de três leguminosas arbóreas nativas do nordeste do Brasil. Archivos Zootecnia, 61:323-334.

Malavolta E, Vitti GC \& Oliveira SA (1997) Avaliação do estado nutricional das plantas. $2^{\mathrm{a}}$ ed. Piracicaba, Associação Brasileira para Pesquisa da Potassa e do Fosfato. 319p.

Oliveira FL, Guerra JGM, Junqueira RM, Silva EE, Oliveira FF, Espíndola JAA, Almeida DL, Ribeiro RLD \& Urquiaga S (2006) Crescimento e produtividade do inhame cultivado entre faixas de guandu em sistema orgânico. Horticultura Brasileira, 24:53-58.

Peoples MB, Palmer B, Lilley DM, Duc LM \& Herridge DF (1996) Application of N-15 and xylem ureide methods for assessing N2 fixation of three shrub legumes periodically pruned for forage. Plant Soil, 182:125-137.

Salmi AP, Guerra JGM, Abboud ACS, Lopes HM \& Martelleto MS (2008) Superação da dormência de sementes de Flemingia macrophylla. Seropédica, Embrapa Agrobiologia. 4p. (Comunicado Técnico, 108).

Unkovich MJ \& Pate JS. (2000) An appraisal of recent field measurements of symbiotic $\mathrm{N}_{2}$ fixation by annual legumes. Field Crops Research, 65:211-228. 